\title{
A mixture of blackberry leaf and fruit extracts decreases fat deposition in HepG2 cells, modifying the gut microbiome
}

\author{
Xuangao Wu ${ }^{1} \cdot$ Bo Ram Jin ${ }^{1} \cdot$ Hye Jeong Yang ${ }^{2}$ Min Jung Kim² $\cdot$ Sunmin Park ${ }^{1}$
}

Received: 12 June 2019 / Accepted: 16 July 2019 / Published Online: 30 September 2019

(C) The Korean Society for Applied Biological Chemistry 2019

\begin{abstract}
More effective treatments are needed for non-alcoholic fatty liver disease (NAFLD). We hypothesized that water extracts of blackberry fruits (BF) and leaves (BL) and their combinations (BFL) reduce fat deposition in HepG2 cells and modulate shortchain fatty acids (SCFA) and fecal bacteria in vitro. HepG2 cells were treated with BF, BL, BFL1:2, and BFL1:3 for $1 \mathrm{~h}$, and 0.5 $\mathrm{mM}$ palmitate was added to the cells. Moreover, low $(30 \mu \mathrm{g} / \mathrm{mL})$ and high doses $(90 \mu \mathrm{g} / \mathrm{mL})$ of $\mathrm{BL}$ and BF were applied to fecal bacteria in vitro, and SCFA was measured by GC. BL, BF, $\mathrm{BFL1} 12$, and BFL1:3 reduced triglyceride deposition in the cells in a dose-dependent manner, and BFL1:2 and BFL1:3 had a stronger effect than BF. The content of malondialdehyde, an index of oxidative stress, was also reduced in BL, BF, and BFL1:2 with increasing superoxide dismutase and glutathione peroxidase activities. The mRNA expression of acetyl CoA carboxylase, fatty acid synthase, and sterol regulatory element-binding protein-1c was reduced in $\mathrm{BL}, \mathrm{BF}, \mathrm{BFL} 1: 2$, and BFL1:3 compared to the control, and BFL1:2 had the strongest effect. By contrast, the carnitine palmitolytransferase-1expression, a regulator of fatty acid oxidation, increased mostly in BFL1:2 and BFL1:3. Tumor necrosis factor- $\alpha$ and interleukin- $1 \beta$ expression was reduced in $\mathrm{BL}$ compared to that in BF and BFL1:2 in HepG2 cells. Interestingly, $\mathrm{BL}$ increased propionate production, and $\mathrm{BF}$ increased butyrate
\end{abstract}

Xuangao Wu and Bo Ram Jin are contributed equally.

Sunmin Park $(\bowtie)$

E-mail: smpark@hoseo.edu

${ }^{1}$ Dept. of Food and Nutrition, Institute of Basic Science, Hoseo University, Asan, Republic of Korea

${ }^{2}$ Food Functional Research Division, Korean Food Research Institutes, Sungnam, Republic of Korea

This is an Open Access article distributed under the terms of the Creative Commons Attribution Non-Commercial License (http://creativecommons. org/licenses/by-nc/3.0/) which permits unrestricted non-commercial use, distribution, and reproduction in any medium, provided the original work is properly cited. and propionate production and increased total SCFA content in fecal incubation. BF increased the contents of Bifidobacteriales and Lactobacillales and decreased those of Clostridiales, whereas BL elevated the contents of Bacteroidales and decreased those of Enterobacteriales. In conclusion, BFL1:2 and BFL1:3 may be potential therapeutic candidates for NAFLD.

Keywords Fatty acid synthesis - Gut microbiome · Nonalcoholic fatty liver disease $\cdot$ Palmitate $\cdot$ Short-chain fatty acids

\section{Introduction}

Fatty liver disease is characterized by an increase in the accumulation of lipid droplets in liver cells due to various conditions such as alcohol intake, high fat diet, and drug administration. It is categorized into alcoholic and non-alcoholic fatty liver disease (NAFLD). Although this condition is reversible, it can progress into hepatitis with inflammation, liver cirrhosis, and liver cancer [1]. The prevalence of NAFLD is rising worldwide (approximately $20-30 \%$ in the USA and $29.6 \%$ in Asia) $[1,2]$. NAFLD is caused by increasing free fatty acids from adipose tissues in the circulation that enter into the liver and damage the hepatic cells by accumulating lipids [3]. The elevation of serum free fatty acid concentrations may be involved in increased insulin resistance. Obesity, dyslipidemia, and hyperglycemia are accompanied by non-alcoholic liver diseases, and obesity with high visceral fat is the major cause of the disease $[4,5]$. Thus, the reduction of insulin resistance is necessary to prevent and treat NAFLD.

Oxidative stress and inflammation increase with elevated insulin resistance, leading to the progression of NAFLD into severe liver diseases. Inflammation is associated with gut microbiomes [6]. Bacteria produce lipopolysaccharide (LPS), metabolites, and short-chain fatty acids (SCFAs). LPS causes low-grade inflammation mediated by the induction of inflammatory cytokines by immune 
cells and adipocytes, and SCFA such as acetate or butyrate can modulate immune cell function [7]. LPS and some SCFAs, especially acetate, can affect adipogenesis and/or insulin resistance $[8,9]$. Thus, the modulation of gut microbiota to produce SCFAs can modulate systemic inflammation and adipogenesis, especially in the gut-liver axis, as the molecules produced in the intestines go to the liver through the portal vein [10].

The medicine market for the treatment of NAFLD is growing and the need for novel treatments for NAFLD is increasing. The ideal treatment agent should enhance lipid utilization in the liver and induce liver cell regeneration. Ursodeoxycholic acid, biphenyl dimethyl dicarboxylate, glutathione, silymarin, and a mixture of flavonolignans extracted from milk thistle (Silybum marianum L. Gaertneri) are used for the treatment for NAFLD [11]. However, more effective therapeutic agents are needed. Blackberry (Rubus alceaefolius) leaves and fruits are reported to decrease insulin resistance, oxidative stress, and inflammation. Anthocyanins, including cianidin-3-glucoside, and total alkaloids in blackberry fruits have a protective effect against non-alcoholic hepatic steatosis in hepatic cells and rats $[12,13]$. Blackberry extracts also have anti-inflammatory activity by modulation of the NF- $\kappa B$ signaling induced non-alcoholic hepatic steatosis in rats [14]. The leaves of blackberry also contain flavonoids, and their water extracts may have an anti-non-alcoholic hepatic steatosis effect.

In the present study, we hypothesized that water extracts of blackberry fruit (BF) and leaves (BL) and their combinations reduce fat deposition in HepG2 cells. Their effects on fecal bacteria were also measured.

\section{Materials and Methods}

\section{Water extract of blackberry leaves and fruits}

Dried Korean blackberry fruits and blackberry leaves (Korea Prime Co., Ltd., Suwon, Korea) were separately powdered and extracted in 20 -fold volumes of $50 \%$ ethanol at $92{ }^{\circ} \mathrm{C}$ for $4 \mathrm{~h}$, and the extracts were concentrated at $40^{\circ} \mathrm{C}$ using a rotary evaporator. The concentrated extracts were lyophilized in a freeze-dryer, and the yield of BF and fruit extracts was 30.8 and $39.0 \%$, respectively.

HPLC was performed using an Ultra-Fast Liquid Chromatography instrument (Shimadzu, Kyoto, Japan) equipped with an autoinjector and a UV detector (Shimadzu SPD-M20A). The extract was analyzed using the Phenomenex Gemini-NX C18 (250 mm×4.6 $\mathrm{mm}$ I.D., $5 \mu \mathrm{m}$ ). The mobile phase was $0.5 \%$ phosphoric acid in water (A) and acetonitrile (B). The gradients were used as follows: $0 \mathrm{~min}, \mathrm{~A}: \mathrm{B}$ 95:5 (v/v); $5 \mathrm{~min}, \mathrm{~A}: \mathrm{B}$ 85:15; $25 \mathrm{~min}, \mathrm{~A}: \mathrm{B}$ 25:80; $35 \mathrm{~min}, \mathrm{~A}: \mathrm{B}$ 0:100; $40 \mathrm{~min}, \mathrm{~A}: \mathrm{B}$ 0:100; $41 \mathrm{~min}, \mathrm{~A}: \mathrm{B} 95: 5$; and $50 \mathrm{~min}, 95: 5$. The mobile phase flow rate was $1.0 \mathrm{~mL} / \mathrm{min}$ under the following conditions: column temperature, $35^{\circ} \mathrm{C}$; injection volume, $20 \mu \mathrm{L}$; and UV detection at $254 \mathrm{~nm}$. The indicator compounds were cyanidin-3-glucoside for BF and ellagic acid for BL. Each indicator compound was purchased from Sigma-Aldrich
(St. Louis, MO, USA) or ChromaDex (Irvine, CA, USA).

\section{Effects of HepG2 cell line on cell damage}

Human hepatocellular carcinoma HepG2 cells were obtained from American Type Culture Collection (HB-8065; Manassas, VA, USA) and were cultured in high-glucose Dulbecco's modified Eagle's medium (DMEM) containing 10\% fetal bovine serum [15]. Cells were transferred into $96-$ well plates at $4 \times 10^{3}$ cells/well in high-glucose DMEM containing $0.3 \%$ bovine serum albumin and allowed to grow to $70 \%$ confluence. The cells were incubated with vehicle, 30 or $90 \mu \mathrm{g} / \mathrm{mL} \mathrm{BF}$ and BL or their mixtures (ratio of $\mathrm{BF}$ and $\mathrm{BL}=1: 2$ (BFL1:2), 1:3 (BFL1:3), and 1:5 (BFL1:5)). After $1 \mathrm{~h}$ of pretreatment with blackberry extracts, $0.5 \mathrm{mM}$ palmitate emulsified with fatty acid free albumin was added to the HepG2 cells, and they were incubated for another $24 \mathrm{~h}$. The cell viability was determined by 3-(4,5-dimethylthiazol-2-yl)-2,5diphenyl tetrazolium bromide (MTT) assay using an Aureon plate reader (Aureon Biosystems, Vienna, Austria).

HepG2 cells were grown in 24 -well plates at $6 \times 10^{4}$ cells/well until the cells were $70 \%$ confluent, then, they were treated with vehicle, 30 or $90 \mu \mathrm{g} / \mathrm{mL} \mathrm{BF}, \mathrm{BL}$, or their mixtures (BFL1:2, BFL1:3, and BFL1:5) for $1 \mathrm{~h}$. The cells were subsequently added with $0.5 \mathrm{mM}$ palmitate and incubated for an additional $24 \mathrm{~h}$, and the cells were lysed with lysis buffer at $4{ }^{\circ} \mathrm{C}$. The cells were used to measure antioxidant and inflammation status and to investigate the mRNA expression of targeted genes.

\section{Fecal bacterial cultivation}

Fecal samples from the large intestine of healthy rats were collected and immediately dissolved in saline containing $1 \% \mathrm{~L}$ cysteine to make $30 \%$ fecal solution. Thioglycolate $0.75 \%$ (BD Medical Technology, 225650, Franklin Lakes, NJ, USA) in phosphate buffer containing $1 \%$ L-cysteine was prepared, autoclaved at $121{ }^{\circ} \mathrm{C}$ for $15 \mathrm{~min}$, and cooled down. The fecal sample $(300 \mu \mathrm{L})$ was added to the $0.75 \%$ thioglycolate medium $(30 \mathrm{~mL})$ for control and supplemented $0.5 \% \mathrm{BL}$ or $\mathrm{BF}$. These processes were conducted in an anaerobic chamber, and the samples were put into the anaerobic jar with an anaerobic pack. The jar was incubated in a shaking bath at $38^{\circ} \mathrm{C}$ at $200 \mathrm{rpm}$ for $48 \mathrm{~h}$. After incubation, the medium was centrifuged, and the bacteria were separated. The medium was used for measuring SCFA, and the bacterial composition was determined by Next Generation Sequencing (NGS).

\section{Oxidative stress and inflammation status}

Lipid peroxide levels in the HepG2 cells treated with $0.5 \mathrm{mM}$ palmitate and $\mathrm{BF}$ and $\mathrm{BL}$ extracts were measured using a thiobarbituric acid reactive substance assay kit (Cayman Chemical, Ann Arbor, MI, USA). The activities of antioxidant enzymes such as $\mathrm{Cu} / \mathrm{Zn}$ superoxide dismutase (SOD) and glutathione (GSH)peroxidase were measured from the lysates of the HepG2 cells by using colorimetry kits (Cayman Chemical, Ann Arbor, MI, USA 
and Biovision, Milpitas, CA, USA, respectively). One unit of each enzyme activity was defined as the amount of SOD needed to inhibit the reaction by $50 \%$, and the enzyme activity was normalized by milligrams of protein in the lysate. GSH level in the liver was also determined using a GSH assay kit (SigmaAldrich, St. Louis, MO, USA).

\section{Isolation of total RNA in HepG2 cells and real-time polymerase chain reaction (PCR) of target genes}

Treated HepG2 cells were mixed with a monophasic solution of phenol and guanidine isothiocyanate (TRIzol reagent; GibcoBRL, Rockville, MD, USA) for total RNA extraction, according to the manufacturer's instructions. cDNA was synthesized from equal amounts of total RNA using SuperScript III reverse transcriptase, and PCR was performed with high fidelity Taq DNA polymerase. Equal amounts of cDNA were added to SYBR Green mix (Bio-Rad, Richmond, CA, USA) and amplified using a real-time PCR instrument (Bio-Rad). The expression levels of the genes of interest were normalized to that of the housekeeping gene $\beta$-actin. To assess changes in the expression of genes related to fatty acid synthesis and oxidation in the liver, the following primers were used: sterol regulatory element-binding protein (SREBP)-1c forward 5'-GGAGCCATGGATTGCACATT-3', reverse 5'-AGGAAGGCTTCCAGAGAGGA-3'; acetyl CoA carboxylase (ACC) forward 5'-GGAAGATGGTGTCCCGCTCTG-3', reverse 3'-GGGGAGATGTGCTGGGTCAT-3'; fatty acid synthase (FAS) forward 5'-AGGTGCTAGAGGCCCTGCTA-3', reverse 5'-GTGC ACAGACACCTTCCCAT-3'; carnitine palmitoyl transferase (CPT)-1 forward 5'-CTCCTGAGCAGTTACCAATGC-3', reverse 5'-GAACCTTGGCTGCGGTAAGAC-3'; TNF- $\alpha$ forward, 5'-AC CCCCAACCTATGAAGAAA-3', reverse 5'-TCCACGCAAAAC GGAATGAA-3'; interleukin (IL)-1 $\beta$ forward, 5'-TTGTGGCTGT GGAGAAGCTG-3', reverse 5'-GCCGTCTTTCATACACAGG3'; $\beta$-actin forward 5'-AAGTCCCTCACCCTCCCAAAAG-3', reverse 5'-AAGCAATGCTGTCACCTTCCC-3'. The primers were designed to sandwich at least one intron in order to distinguish between the products derived from mRNA and genomic DNA. At least four PCR reactions per group were performed as previously described [16].

\section{Gas chromatography for measuring SCFA}

After incubation of rat fecal samples, the supernatants of the media were mixed with ethanol (1:2), and $5 \mathrm{M} \mathrm{HCl}$ was added to reach $\mathrm{pH} 2$. The mixture was centrifuged at $4{ }^{\circ} \mathrm{C}$ at $2,000 \times \mathrm{g}$ for $15 \mathrm{~min}$, and the supernatant was filtered with a $0.45-\mu \mathrm{m}$ syringe filer. The filtrate was injected into a gas chromatograph (PerkinElmer, Waltham, MA, USA) using a fused-silica capillary column $(30 \mathrm{~m} \times 0.25 \mathrm{~mm})$ coated with a 0.25 -ìm-thick free fatty acid phase film (DB_FFAP 122-3232, J\&W Scientific, Agilent Technologies Inc., Santa Clara, CA, USA). Gas chromatography was conducted. Helium was supplied as the carrier gas using a linear velocity mode at $22.2 \mathrm{~cm} \mathrm{sec}^{-1}$. Glass wool was inserted in the liner of the splitless injection port at $230{ }^{\circ} \mathrm{C}$. The initial oven temperature was set at $100{ }^{\circ} \mathrm{C}$, then gradually increased at $10 \mathrm{~min}^{-1}$ to 180,20 $\min ^{-1}$ to $220^{\circ} \mathrm{C}$, and maintained at $220^{\circ} \mathrm{C}$ for $8 \mathrm{~min}$. The FID detector was used, and the temperature was $240^{\circ} \mathrm{C}$. Flow rates were 40,400 , and $80 \mathrm{~mL} \mathrm{~min}{ }^{-1}$ for hydrogen, air, and helium, respectively.

\section{Gut microbiome measured by NGS}

The gut microbiome composition was measured from culture rat feces by analyzing metagenome sequencing using NGS. Bacterial DNA was extracted from the samples of each rat using a Power Fecal DNA Isolation kit (MoBio, Carlsbad, CA, USA) according to the manufacturer's instructions. Each library was prepared using PCR products according to the GS FLX plus library prep guide. The emPCR, corresponding to clonal amplification of the purified library, was carried out using the GS FLX plus emPCR kit (454 Life Sciences, Branford, CT, USA). Libraries were immobilized onto DNA capture beads. The library beads were added to the amplification mix and the oil, and the mixture was vigorously shaken on a Tissue Lyser II (Qiagen, Valencia, CA, USA) to create micro-reactors containing both amplification mix and a single bead. The emulsion was dispensed into a 96-well plate, and the PCR amplification program was run with $16 \mathrm{~S}$ universal primers in the Fast Start High Fidelity PCR System (Roche, Basel, Switzerland) according to the manufacturer's recommendations. Sequencing of bacterial DNA in the feces was performed by the Macrogen Ltd. (Seoul, Korea) using a Genome Sequencer GS FLX plus (454 Life Sciences) as previously reported [17]. The 16S amplicon sequences were processed using Mothur v.1.36. We followed the Miseq SOP to identify the taxonomy and count the bacteria in each fecal sample. The selection of operational taxonomic units was delimited at $98 \%$ identity, which was taxonomically classified by consensus using Greengenes 13_8_99.

\section{Statistical analysis}

Statistical analysis was performed using SAS, version 7.0. Results are expressed as means \pm standard deviations. The differently treated groups were compared by one-way analysis of variance. Significant differences between groups were identified by Tukey's tests at $p<0.05$.

\section{Results}

The contents of cyanidin-3-glucoside and ellagic acid, index compounds

The $50 \%$ ethanol extracts of BF and BL contained $12 \pm 0.4 \mathrm{mg}$ cyanidin-3-glucoside and $28.3 \pm 1.2 \mathrm{mg}$ ellagic acid, respectively ( $\mathrm{n}=3$; Fig. 1). 
A

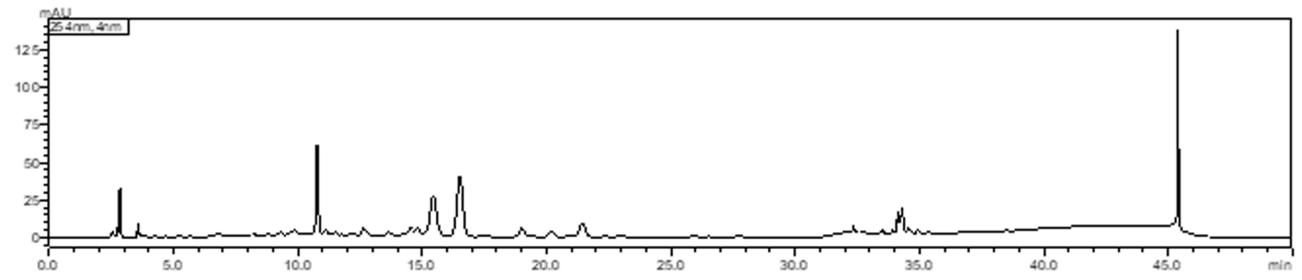

B

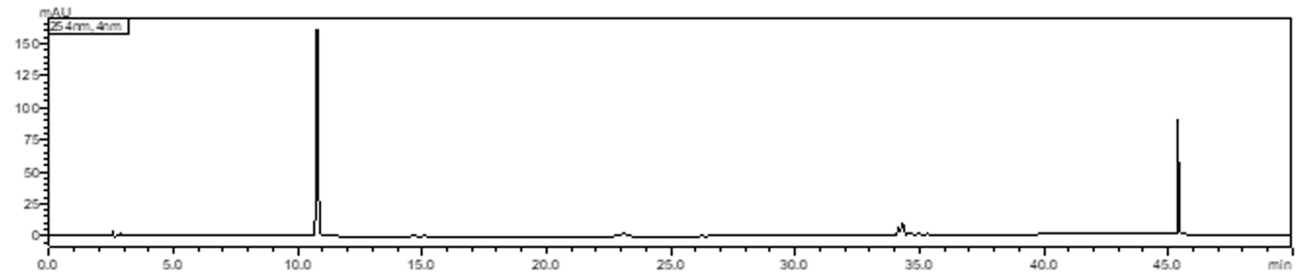

C

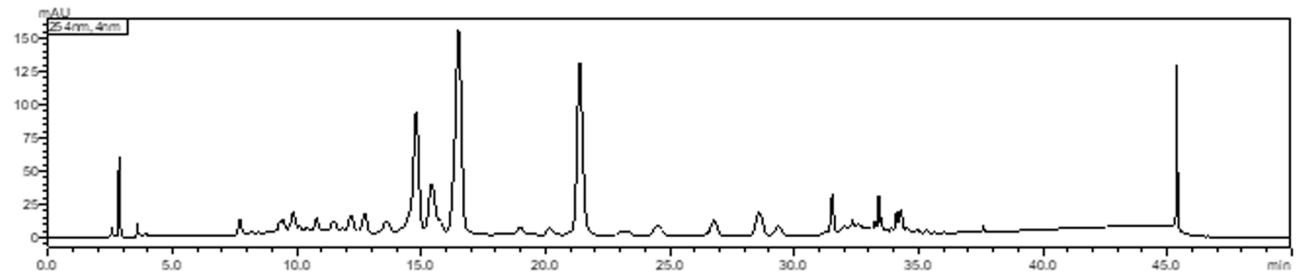

D

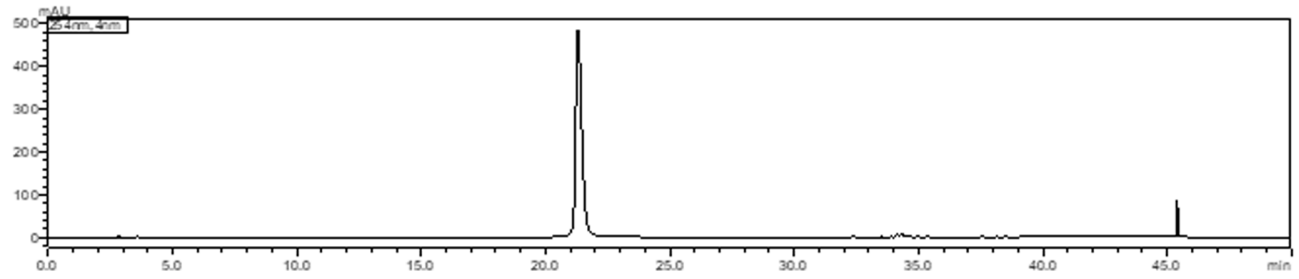

Fig. 1 HPLC chromatogram of 50\% ethanol extracts of blackberry. (A) fruit and leaf (B) Blackberry fruit (C) Standard of cyanidin-3-glucoside (D) Blackberry leaves (E) Standard of ellagic acid

\section{Improvement of cell viability by BFL1:2 and BFL1:3}

Palmitate administration $(0.5 \mathrm{mM})$ inhibited cell viability in HepG2 cells, and BL and BF dose-dependently protected against cell death by palmitate intoxication (Fig. 2A). High dosage of BL (30 and $90 \mu \mathrm{g} / \mathrm{mL}$ ) showed greater protective activity than that of BF (Fig. 2A). BFL1:2, BFL1:3, BFL1:4, and BFL1:5 improved cell viability dose-dependently, and BFL1:2 and BFL1:3 were the most effective in protecting against cell damage (Fig. 2B). However, the improvement was not as much as the normal control group in HepG2 cells (Fig. 2B).

Decrease of TG deposition and mRNA expression of genes related fatty acid synthesis by BFL1:2 and BFL1:3

Palmitate pretreatment increased TG accumulation in HepG2 cells. BL, BF, and BFL inhibited TG deposition in a dosedependent manner, and $\mathrm{BL}$ decreased TG deposition more than BF. The high dosage of BFL1:2 and BFL1:3 was the most effective in suppressing TG accumulation (Fig. 3A). The mRNA expression of ACC, FAS, and SREBP-1c was involved in the fatty acid synthesis, and the mRNA expression of CPT-1 was associated with fatty acid oxidation in HepG2 cells (Fig. 3B). The mRNA expression of $\mathrm{ACC}$ was reduced in the normal control compared to the control, and BL, BF, BFL1:2, and BFL1:3 also decreased.
The decrease associated with BF was smaller than that associated with BL. The mRNA expression of FAS and SREBP-1c exhibited similar patterns under different treatments (Fig. 3A). The mRNA expressions of FAS and SREBP-1c were reduced in a descending order of the control, BF, BL, BFL1:3, BFL1:2, and normal control. The mRNA expression of CPT-1 was opposite to that of FAS, and it was lower in the control than in the normal control. The CPT-1 mRNA expression increased in ascending order of the control, BF, BL, BFL1:2, BFL1:3 and normal control (Fig. 3B).

\section{Reduction of oxidative stress and inflammation by BFL1:2 and BFL1:3}

The cell damage by palmitate administration was associated with increased oxidative stress and inflammation. The MDA levels representing lipid peroxide deposition were higher by approximately 2.5 folds in the HepG2 cells damaged with palmitate (control) compared to those in the normal control (Fig. 4A). MDA contents in HepG2 cells increased in the control compared to the normal control, and BL and BF suppressed the MDA increase from the control. BFL1:2 $(90 \mu \mathrm{g} / \mathrm{mL})$ inhibited MDA contents the most. SOD and GSH-Px levels were reduced in the control compared to the normal control (Fig. 4A). BF treatment $(90 \mu \mathrm{g} / \mathrm{mL})$ increased SOD level more than BL, but GSH-Px activity was elevated in BL 

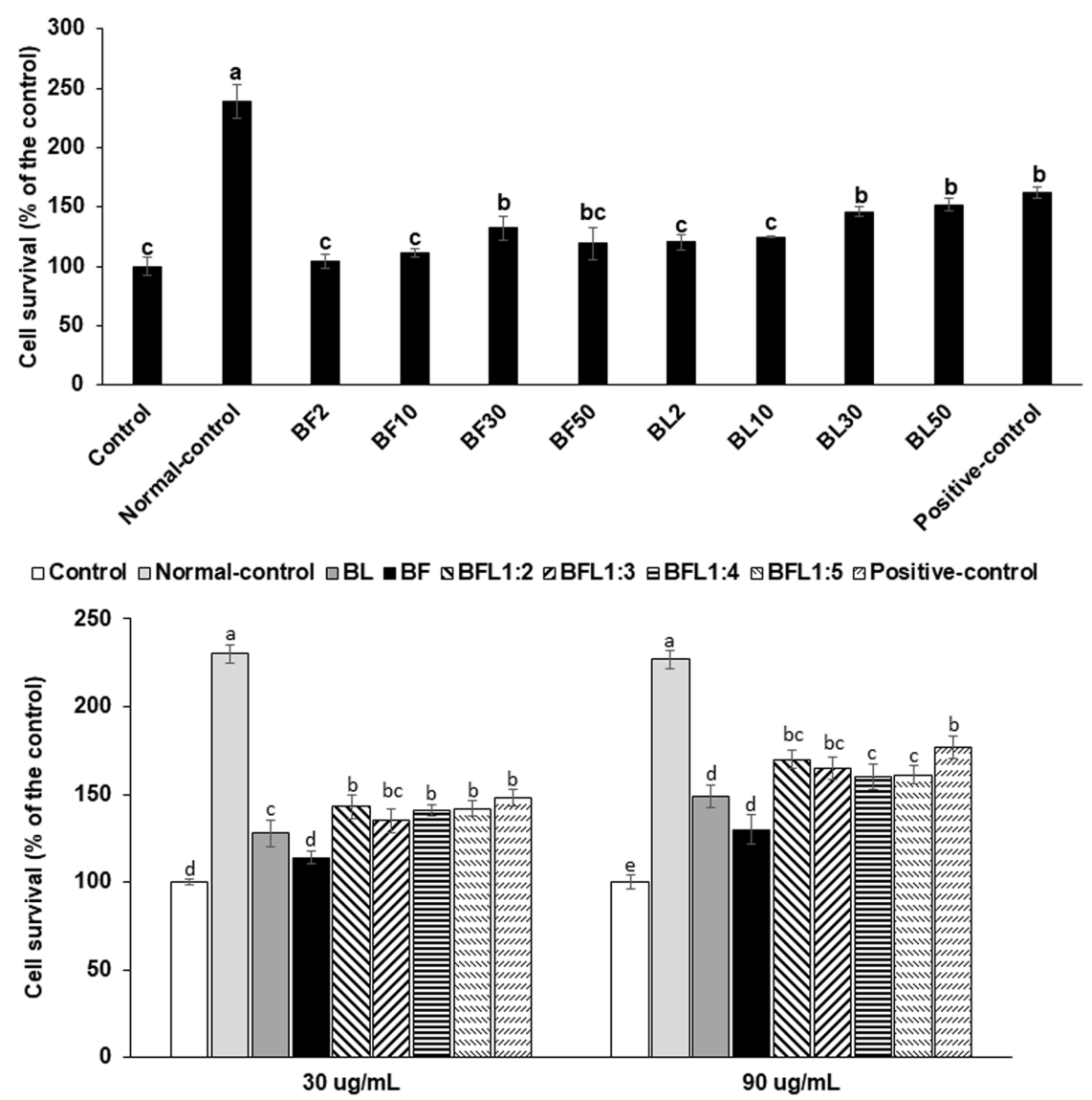

Fig. 2 Cell survival in palmitate (PA)-induced cell damaged HepG2 cells treated with blackberry fruit and leaf extracts. Cell survival measured by MTT at different dosages of $50 \%$ ethanol extracts of blackberry leaf (BL) and fruit (BF) in $0.5 \mathrm{mM}$ PA-treated HepG2 cells. Cell survival measured by MTT in $0.5 \mathrm{mM}$ PA-treated HepG2 cells treated with 30 and $90 \mu \mathrm{g}$ extracts of blackberry leaves and fruits per milliliter of medium .Control, $0.5 \mathrm{mM}$ PA plus vehicle; Normal-control, vehicle; BF2, BF10, BF30 and BF50, $0.5 \mathrm{mM}$ PA plus 2, 10, 30, and $50 \mu \mathrm{g} / \mathrm{mL}$ BF, respectively; BL2, BL10, BL30 and BL50, $0.5 \mathrm{mM}$ PA plus 2, 10, 30, and $50 \mu \mathrm{g} / \mathrm{mL}$ BL. ${ }^{\text {a,b,c,d,e }}$ Different letters on the bars represent significant differences in each parameter at $p<0.05$

and BF. BFL1:2 increased SOD and GSH-Px activities the most, but the activities in BFL1:2 were lower than that in the normal control (Fig. 4A).

In contrast to the activities of antioxidant enzymes, the mRNA expression of proinflammatory cytokines was elevated in the control group compared to the normal control (Fig. 4B). The mRNA expression of TNF- $\alpha$ and IL- $1 \beta$ decreased in BL, BF, BFL1:2, and BFL1:3 groups compared to the control, and it was the lowest in BFL1:2 and BFL1:3 groups. Especially, TNF- $\alpha$ expression in BFL1:2 and BFL1:3 groups decreased as much as that in the normal control group (Fig. 4B).

Increase of propionic acid and butyric acid by BF and BL, respectively, in fecal culture

Total production of SCFA was highest in the group treated with $\mathrm{BF}$, followed by BL and then control (Fig. 5A). BF increased butyric acid and propionic acid the most, whereas BL increased propionic acid more than the control (Fig. 5A).
Increase of Bifidobacteriales by BF and Bacteroidales by BL As shown in Fig. 5B, BF increased Bifidobacteriales and Coriobacteriales but decreased Bacteroidales compared to the control. BL increased Bacteroidales (2.5 folds) and Fibrobacterales compared to the control. Lactobacillales did not differ between $\mathrm{BF}$ and control, but they decreased in BL. BF decreased Clostridiales compared to the control. Enterobacteriales, harmful bacteria, decreased in both $\mathrm{BL}$ and $\mathrm{BF}$ compared to the control, and they were lower in $\mathrm{BL}$ than in BF. Thus, BL and BF differently influenced gut microbiome composition, but both improved gut microbiome composition compared to the control.

\section{Discussion}

Although the incidence of NAFLD is increasing, preventive and therapeutic measures are not sufficient. NAFLD results in increased hepatic fat deposition, and it accompanied by inflammation that 

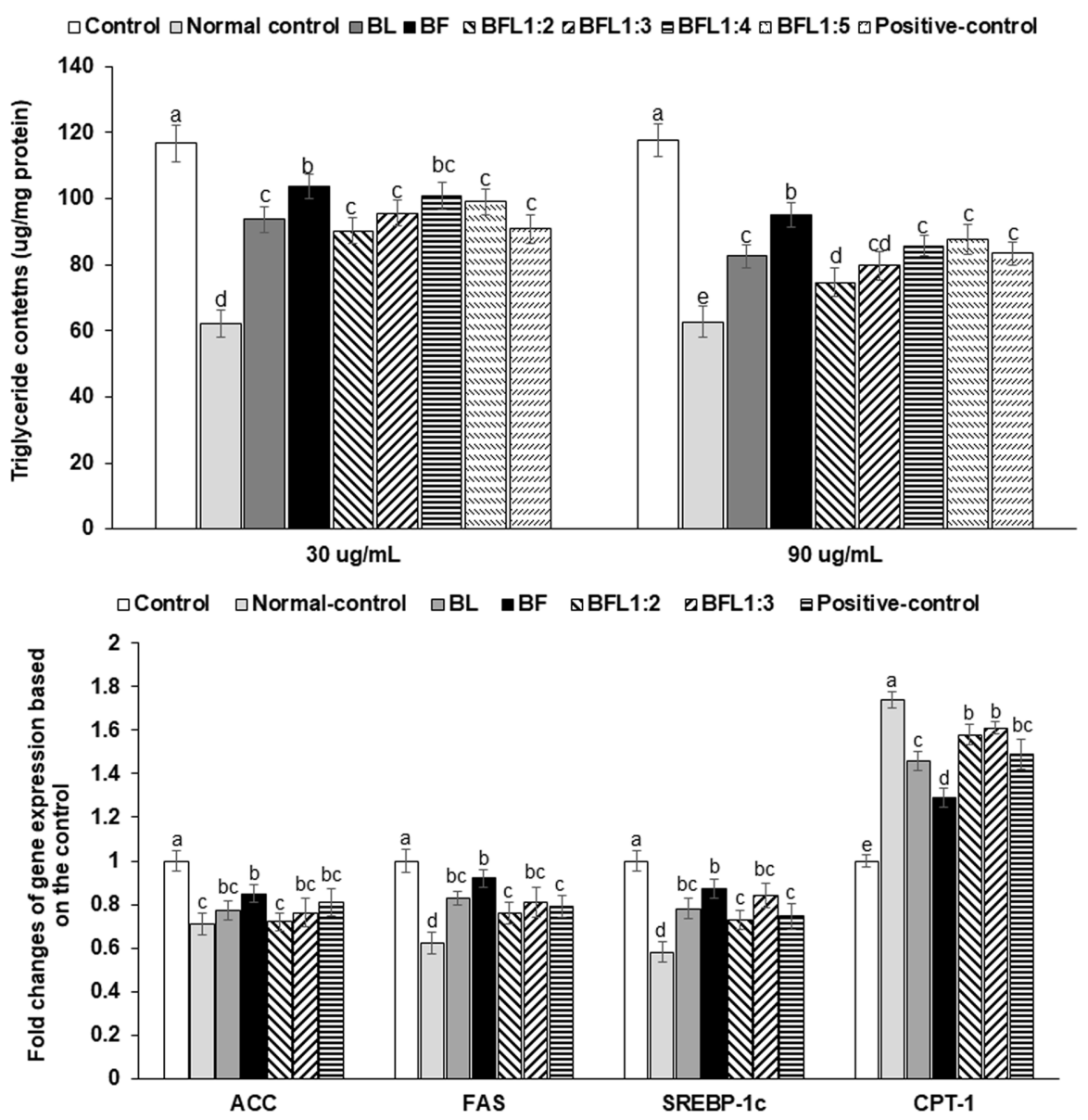

Fig. 3 Triglyceride contents in palmitate (PA)-induced cell damaged HepG2 cells treated with blackberry fruit and leaf extracts. Triglyceride contents in $0.5 \mathrm{mM}$ PA-treated HepG2 cells treated with 30 and $90 \mu \mathrm{g}$ extracts of blackberry leaves and fruits per milliliter of medium. Fold differences in mRNA expression of genes related to fatty acid synthesis and oxidation in $0.5 \mathrm{mM}$ PA-treated HepG2 cells treated with $90 \mu \mathrm{g}$ extracts of blackberry leaves and fruits per milliliter of medium. Control, $0.5 \mathrm{mM}$ PA plus vehicle; Normal-control, vehicle; BL, BF, BFL1:2, BFL1:3, BFL1:4 and BFL1:5, 0.5 mM PA plus 30 or $90 \mathrm{ug} / \mathrm{mL}$ BL, BF, BFL1:2, BFL1:3, BFL1:4 and BFL1:5, respectively. BFL1:2 indicated the mixture of BF 1 portion and BL 2 portion. a,b,c,d,e Different letters on the bars represent significant differences in each parameter at $p<0.05$

progressed into non-alcoholic steatohepatitis. Excessive energy intake through a high fat diet is the major cause of these diseases, although this does not completely account for NAFLD induction. It is accompanied by oxidative stress and inflammation. Anthocyanins and flavonoids are well-known to reduce fatty acid synthesis, oxidative stress, and inflammation. Silymarin, a flavonoid, is used for treating NAFLD. In the present study, we determined whether $\mathrm{BF}$ and $\mathrm{BL}$ and their combinations (BFL) could be a potential treatment for NAFLD. BL and BF reduced fat deposition in HepG2 cells compared to the control, and BL was more effective than BF. BL reduced mRNA expression of genes related to fatty acid synthesis and increased mRNA expression of genes related to fatty acid oxidation compared to BF. Interestingly, the mixture of $\mathrm{BF}$ and $\mathrm{BL}$ (1:2 and 1:3) decreased mRNA expression more than individual extracts. Lipid peroxide contents were lowered in $\mathrm{BF}$ and BL than the control. BFL1:2 reduced the lipid peroxide contents compared to individual extracts. $\mathrm{BL}$ and $\mathrm{BF}$ increased
SCFA compared to the control and modulated the composition of fecal bacteria in vitro. Therefore, BL, BFL1:2, and BFL1:3 may have a therapeutic activity for NAFLD, and further experiments in animals and humans are needed.

NAFLD is associated with excessive triglyceride deposition, with increased oxidative stress and inflammation. In cell-based studies, palmitate administration develops excessive triglyceride deposition, showing characteristics similar to NAFLD. Increased serum concentrations of free fatty acids are the major cause of NAFLD by stimulate fat deposition in the liver in experimental animals and humans. Berry fruits and leaves have anthocyanins and flavonoids that are involved in reducing oxidative stress and inflammation in vitro and in vivo $[18,19]$. Berries, including cranberry, blackberry, wild blueberry, strawberry, and chokeberry have antioxidant activities in HepG2 cells in oleic acid-induced hepatic steatosis [13]. The major index compound of blackberry and blueberry is a cyanidin-3-glucoside that improves metabolic 

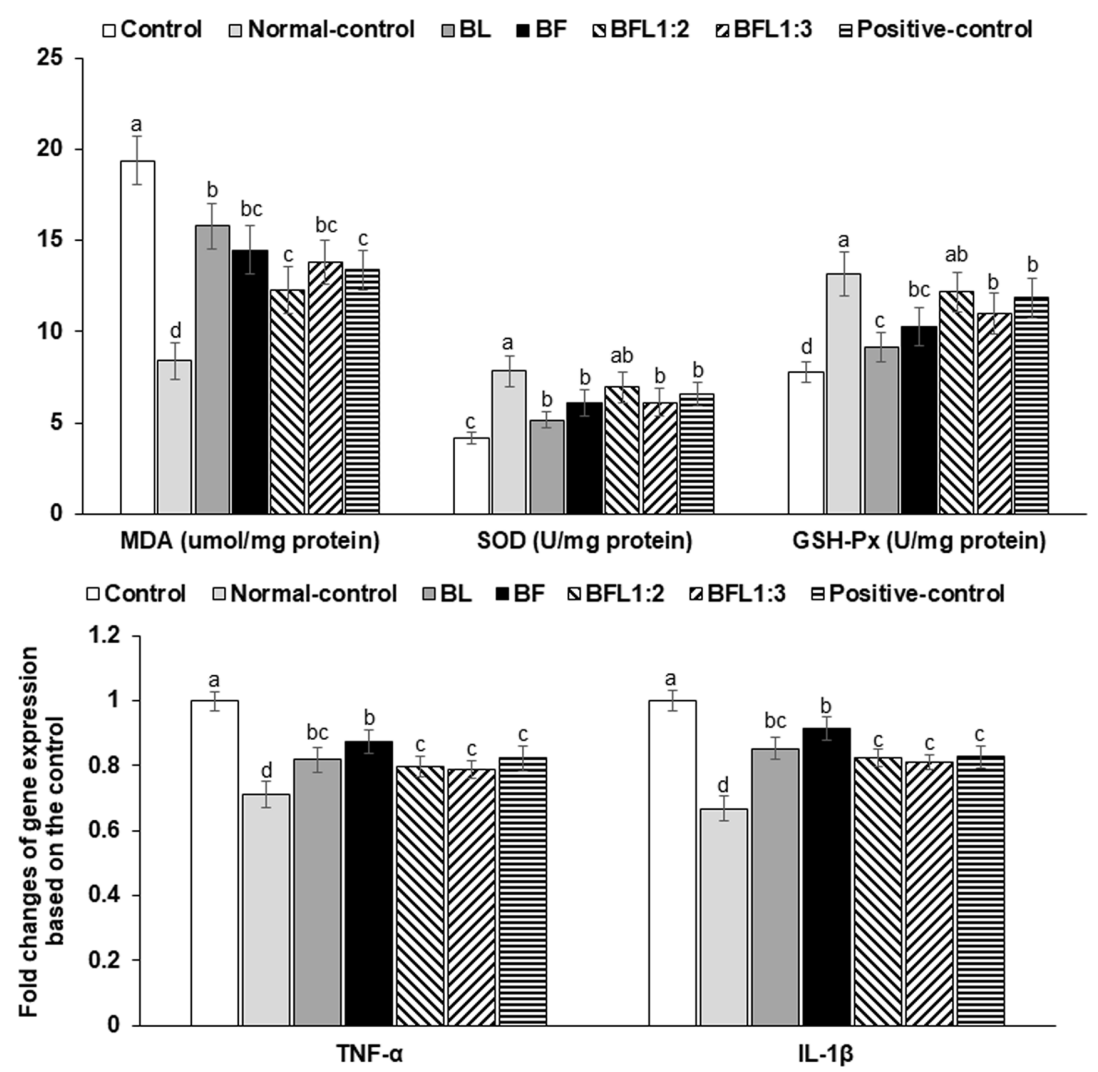

Fig. 4 Lipid peroxide and antioxidant enzyme activities in palmitate (PA)-induced cell damaged HepG2 cells treated with blackberry fruit and leaf extracts. Malondialdehyde (MDA) contents and antioxidant enzyme activities, including superoxide dismutase (SOD) and glutathione peroxidase (GSH-x) after treating $90 \mu \mathrm{g}$ extracts of blackberry leaves and fruits per milliliter of medium. The mRNA expression of genes related to proinflammatory cytokines, including tumor necrosis factor (TNF)- $\alpha$ and interleukin (IL)-1â after treating $90 \mu \mathrm{g}$ extracts of blackberry leaves and fruits per milliliter of medium. Control, 0.5 mM PA plus vehicle; Normal-control, vehicle; BL, BF, BFL1:2, BFL1:3, BFL1:4 and BFL1:5, 0.5 mM PA plus 30 or $90 \mathrm{ug} / \mathrm{mL}$ BL, BF, BFL1:2, BFL1:3, BFL1:4 and BFL1:5, respectively. BFL1:2 indicated the mixture of BF 1 portion and BL 2 portion. a,b,c,d Different letters on the bars represent significant differences in each parameter at $p<0.05$

disorders, including NAFLD, and increases the infiltration of inflammatory markers in the liver [20-22]. Ellagic acid in berries is a natural antioxidant polyphenol and alleviates NAFLD by mitigating hepatic oxidative stress and insulin resistance in type 2 diabetic female rats $[23,24]$. Thus, BL and BF can reduce fat deposition in the liver to attenuate NAFLD.

The gut microbiome is also involved in NAFLD [25]. High fat diet can cause intestinal dysbiosis by modulating the production of substances such as SCFA, bile acids, and bacterial components and by activating toll-like receptors [26]. Some substances including acetate increase insulin resistance and inflammation and induce intestinal permeability due to malfunction of tight junctions. However, butyrate-producing bacteria reduces NAFLD progression $[27,28]$. Thus, the dysbiosis of gut microbiome in a high fat diet is possible mechanism to develop NAFLD. Polyphenols, including anthocyanins and flavonoids, show low bioavailability [29]. Polyphenol biotransformation by colonic fermentation improves bioavailability to exert health benefits [29,30]. Digested and fermented phenolic compounds in blackberry by gut microbiota fermentation ameliorate palmitate-induced lipid deposition in HepG2 cells [31]. The present study showed that the composition of intestinal bacteria and SCFA was differently changed by BL and BF extracts. The modulation may affect the fat deposition in the liver by altering the gut-liver axis. Gowd et al. [31] have demonstrated that gut metabolites of blackberry significantly elevate the glucose uptake and glycogen content in HepG2 cells. Gut metabolites also ameliorate high-glucose plus palmitiateinduced oxidative stress by reducing reactive oxygen species overproduction and glutathione depletion in HepG2 cells. The present study also showed that $\mathrm{BL}, \mathrm{BF}$, and the mixtures of $\mathrm{BL}$ and $\mathrm{BF}$ decreased fat deposition and oxidative stress. BFL1:2 showed the most beneficial activity in reducing fat accumulation, oxidative stress, and inflammation in HepG2 cells.

In conclusion, both $\mathrm{BL}$ and $\mathrm{BF}$ decreased triglyceride deposition, and their mixture (BFL1:2) reduced it the most in HepG2 cells. This was associated with an increase in fatty acid synthesis and a decrease in fatty acid oxidation. BF reduced oxidative stress better than BL, and BFL1:2 decreased oxidative stress the most by 

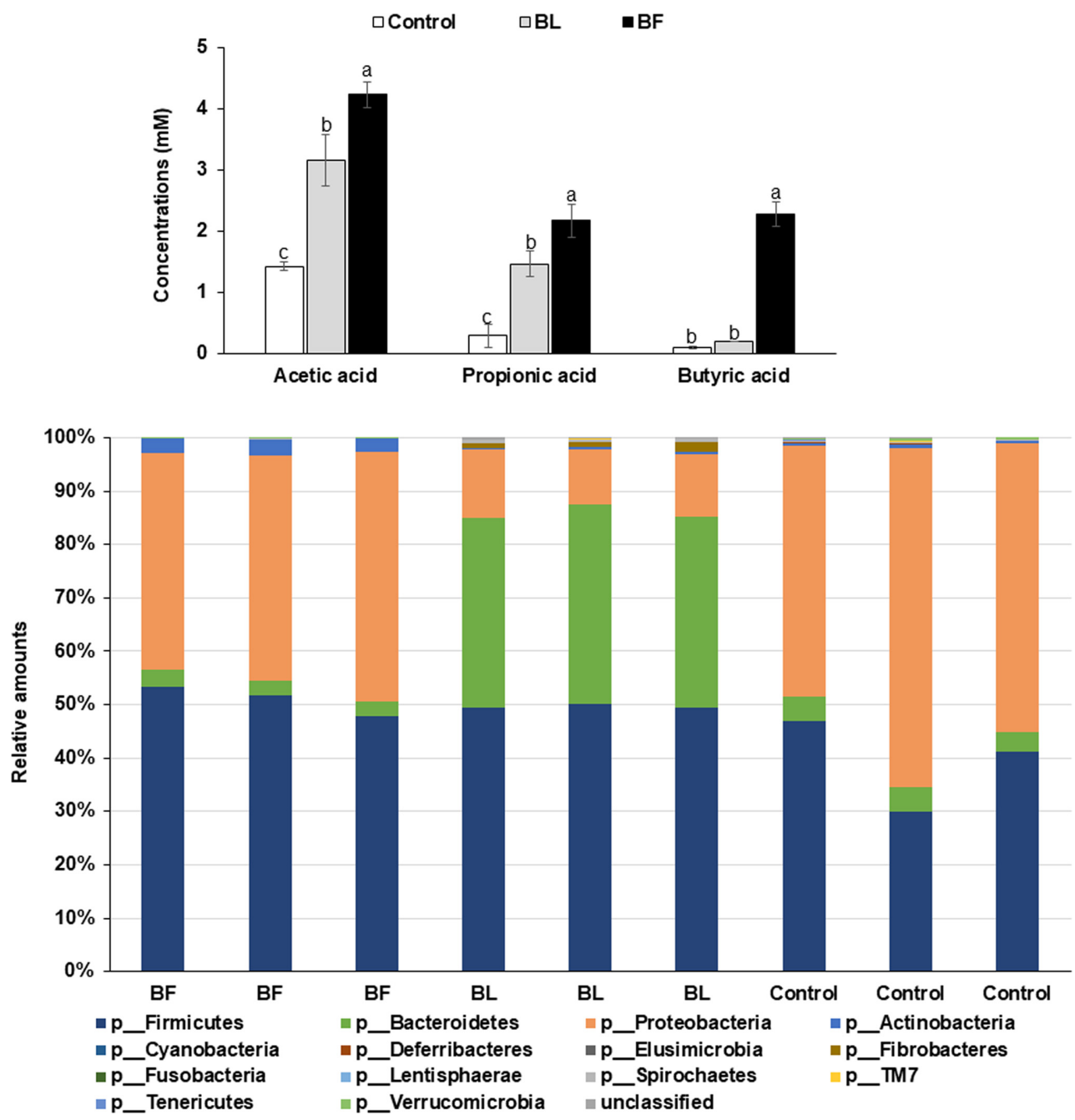

Fig. 5 Short-chain fatty acid contents and bacterial composition. Contents of short-chain fatty acids after incubating rat feces with $90 \mu \mathrm{g}$ extracts of blackberry leaves and fruits per milliliter of medium measured by GC. Composition of fecal microbiome. Control, vehicle; BL and BF, $0.5 \% \mathrm{BL}$ and $\mathrm{BF}$ treatment in the culture media, respectively. ${ }^{\mathrm{ab}, \mathrm{c}}$ Different letters on the bars represent significant differences in each parameter at $p<0.05$

elevating the activities of antioxidant enzymes. BL and BF increased SCFA and altered the gut microbiome composition, increasing their effect on NAFLD. Thus, BFL1:2 may be beneficial to prevent or alleviate NAFLD. Further animal and human studies are needed to confirm these benefits.

Acknowledgments This study was funded by the Ministry of Food, Agriculture, Forestry, and Fisheries and the Korea Science and Engineering Foundation, 2018.

Authors' contributions SP designed the studies, analyzed the data and wrote the paper. HJY and $\mathrm{XW}$ conducted the measurement of index compounds. XW and BRJ performed gut microbiome and HepG2 cell experiments.

Competing interests The authors declare that they have no competing interests.
Open Access This article is distributed under the terms of the Creative Commons Attribution 4.0 International License (http://creativecommons.org/ licenses/by/4.0/), which permits unrestricted use, distribution, and reproduction in any medium, provided you give appropriate credit to the original author(s) and the source, provide a link to the Creative Commons license, and indicate if changes were made.

Publisher's Note Springer Nature remains neutral with regard to jurisdictional claims in published maps and institutional affiliations.

\section{References}

1. Younossi Z, Anstee QM, Marietti M, Hardy T, Henry L, Eslam M, George J, Bugianesi E (2018) Global burden of NAFLD and NASH: trends, predictions, risk factors and prevention. Nat Rev Gastroenterol Hepatol 15: 11-20

2. Li J, Zou B, Yeo YH, Feng Y, Xie X, Lee DH, Fujii H, Wu Y, Kam LY, 
Ji F, Li X, Chien N, Wei M, Ogawa E, Zhao C, Wu X, Stave CD, Henry L, Barnett S, Takahashi H, Furusyo N, Eguchi Y, Hsu YC, Lee TY, Ren W, Qin C, Jun DW, Toyoda H, Wong VW, Cheung R, Zhu Q, Nguyen MH (2019) Prevalence, incidence, and outcome of non-alcoholic fatty liver disease in Asia, 1999-2019: a systematic review and meta-analysis. Lancet Gastroenterol Hepatol 4: 389-398

3. Buzzetti E, Pinzani M, Tsochatzis EA (2016) The multiple-hit pathogenesis of non-alcoholic fatty liver disease (NAFLD). Metabolism 65: $1038-1048$

4. Katsiki N, Mikhailidis DP, Mantzoros CS (2016) Non-alcoholic fatty liver disease and dyslipidemia: An update. Metabolism 65: 1109-1123

5. Lallukka S, Yki-Jarvinen H (2016) Non-alcoholic fatty liver disease and risk of type 2 diabetes. Best Pract Res Clin Endocrinol Metab 30: 385 395

6. Doulberis M, Kotronis G, Gialamprinou D, Kountouras J, Katsinelos P (2017) Non-alcoholic fatty liver disease: An update with special focus on the role of gut microbiota. Metabolism 71: 182-197

7. Mokhtari Z, Gibson DL, Hekmatdoost A (2017) Nonalcoholic Fatty Liver Disease, the Gut Microbiome, and Diet. Adv Nutr 8: 240-252

8. Ivan J, Major E, Sipos A, Kovacs K, Horvath D, Tamas I, Bay P, Dombrádi V, Lontay B (2017) The Short-Chain Fatty Acid Propionate Inhibits Adipogenic Differentiation of Human Chorion-Derived Mesenchymal Stem Cells Through the Free Fatty Acid Receptor 2. Stem Cells Dev 26: 1724-1733

9. Yu H, Li R, Huang H, Yao R, Shen S (2018) Short-Chain Fatty Acids Enhance the Lipid Accumulation of 3T3-L1 Cells by Modulating the Expression of Enzymes of Fatty Acid Metabolism. Lipids 53: 77-84

10. Wiest R, Albillos A, Trauner M, Bajaj JS, Jalan R (2017) Targeting the gut-liver axis in liver disease. J Hepatol 67: 1084-1103

11. Zhong S, Fan Y, Yan Q, Fan X, Wu B, Han Y, Zhang Y, Chen Y, Zhang $\mathrm{H}$, Niu J (2017) The therapeutic effect of silymarin in the treatment of nonalcoholic fatty disease: A meta-analysis (PRISMA) of randomized control trials. Medicine (Baltimore) 96: e9061

12. Zheng H, Zhao J, Zheng Y, Wu J, Liu Y, Peng J, Hong Z (2014) Protective effects and mechanisms of total alkaloids of Rubus alceaefolius Poir on nonalcoholic fatty liver disease in rats. Mol Med Rep 10: 1758-1764

13. Wang Y, Zhao L, Wang D, Huo Y, Ji B (2016) Anthocyanin-rich extracts from blackberry, wild blueberry, strawberry, and chokeberry: antioxidant activity and inhibitory effect on oleic acid-induced hepatic steatosis in vitro. J Sci Food Agric 96: 2494-2503

14. Zhao J, Zheng H, Liu Y, Lin J, Zhong X, Xu W, Hong Z, Peng J (2013) Anti-inflammatory effects of total alkaloids from Rubus alceifolius Poir [corrected]. on non-alcoholic fatty liver disease through regulation of the NF-kappaB pathway. Int J Mol Med 31: 931-937

15. Lopez-Terrada D, Cheung SW, Finegold MJ, Knowles BB (2009) Hep G2 is a hepatoblastoma-derived cell line. Hum Pathol 40: 1512-1515

16. Park S, Kim DS, Wu X, Q JY (2018) Mulberry and dandelion water extracts prevent alcohol-induced steatosis with alleviating gut microbiome dysbiosis. Exp Biol Med (Maywood) 243: 882-894

17. Moon NR, Kang S, Park S (2018) Consumption of ellagic acid and dihydromyricetin synergistically protects against UV-B induced photoaging, possibly by activating both TGF-betal and wnt signaling pathways. J Photochem Photobiol B 178: 92-100

18. Carvalho MMF, Reis LLT, Lopes JMM, Lage NN, Guerra J, Zago HP, Bonomo LF, Pereira RR, Lima WG, Silva ME, Pedrosa ML (2018) Acai improves non-alcoholic fatty liver disease (NAFLD) induced by fructose. Nutr Hosp 35: 318-325

19. Ren T, Huang C, Cheng M (2014) Dietary blueberry and bifidobacteria attenuate nonalcoholic fatty liver disease in rats by affecting SIRT1mediated signaling pathway. Oxid Med Cell Longev 2014: 469059

20. Bhaswant M, Fanning K, Netzel M, Mathai ML, Panchal SK, Brown L (2015) Cyanidin 3-glucoside improves diet-induced metabolic syndrome in rats. Pharmacol Res 102: 208-217

21. Pei L, Wan T, Wang S, Ye M, Qiu Y, Jiang R, Pang N, Huang Y, Zhou Y, Jiang X, Ling W, Zhang Z, Yang L (2018) Cyanidin-3-O-beta-glucoside regulates the activation and the secretion of adipokines from brown adipose tissue and alleviates diet induced fatty liver. Biomed Pharmacother 105: 625-632

22. You Y, Yuan X, Liu X, Liang C, Meng M, Huang Y, Han X, Guo J, Guo Y, Ren C, Zhang Q, Sun X, Ma T, Liu G, Jin W, Huang W, Zhan J (2017) Cyanidin-3-glucoside increases whole body energy metabolism by upregulating brown adipose tissue mitochondrial function. Mol Nutr Food Res 61

23. Polce SA, Burke C, Franca LM, Kramer B, de Andrade Paes AM, Carrillo-Sepulveda MA (2018) Ellagic Acid Alleviates Hepatic Oxidative Stress and Insulin Resistance in Diabetic Female Rats. Nutrients 10

24. Panchal SK, Ward L, Brown L (2013) Ellagic acid attenuates highcarbohydrate, high-fat diet-induced metabolic syndrome in rats. Eur J Nutr 52: 559-568

25. Koukias N, Buzzetti E, Tsochatzis EA (2017) Intestinal hormones, gut microbiota and non-alcoholic fatty liver disease. Minerva Endocrinol 42: 184-194

26. Cui Y, Wang Q, Chang R, Zhou X, Xu C (2019) Intestinal Barrier Function-Non-alcoholic Fatty Liver Disease Interactions and Possible Role of Gut Microbiota. J Agric Food Chem 67: 2754-2762

27. Endo H, Niioka M, Kobayashi N, Tanaka M, Watanabe T (2013) Butyrate-producing probiotics reduce nonalcoholic fatty liver disease progression in rats: new insight into the probiotics for the gut-liver axis. PLoS One 8: e63388

28. Perry RJ, Peng L, Barry NA, Cline GW, Zhang D, Cardone RL, Petersen KF, Kibbey RG, Goodman AL, Shulman GI (2016) Acetate mediates a microbiome-brain-beta-cell axis to promote metabolic syndrome. Nature 534: $213-217$

29. Chen L, Cao H, Xiao J (2018) 2-Polyphenols: Absorption, bioavailability, and metabolomics. In: Galanakis CM (ed) Polyphenols: Properties, Recovery, and Applications. Woodhead Publishing, pp 45-67

30. Frolinger T, Sims S, Smith C, Wang J, Cheng H, Faith J, Ho L, Hao K, Pasinetti GM (2019) The gut microbiota composition affects dietary polyphenols-mediated cognitive resilience in mice by modulating the bioavailability of phenolic acids. Sci Rep 9: 3546

31. Gowd V, Bao T, Wang L, Huang Y, Chen S, Zheng X, Cui S, Chen W (2018) Antioxidant and antidiabetic activity of blackberry after gastrointestinal digestion and human gut microbiota fermentation. Food Chem 269: 618-627 